\title{
The tuberous sclerosis complex-associated giant renal angiomyolipoma: A case report
}

\author{
PENGFEI SUN, SHUANGXING CHEN, ZHENGMING SU and YONGZHONG HE \\ Department of Urology, The Fifth Affiliated Hospital of Guangzhou Medical University, \\ Guangzhou, Guangdong 510700, P.R. China
}

Received April 28, 2020; Accepted October 1, 2020

DOI: $10.3892 / \mathrm{mco} .2021 .2214$

\begin{abstract}
Renal angiomyolipoma (RAML), also referred to as renal hamartoma, is a rare benign tumor. There are two types of RAML, which include the tuberous sclerosis complex (TSC)-associated type and the sporadic type. TSC is an autosomal dominant genetic disease characterized by the growth of benign tumors in the skin, brain, kidneys, lung and heart. TSC leads to organ dysfunction, as the normal parenchyma is replaced by a variety of cell types. The current study presents a case of giant RAML in a 20 -year-old female, who was hospitalized for epileptic seizures. Large abdominal lesions were detected during hospitalization. Subsequently, she underwent open mass resection and right kidney partial resection. Postoperative pathological examination confirmed that the mass was angiomyolipoma.
\end{abstract}

\section{Introduction}

Renal angiomyolipoma (RAML) is among the most common kidney benign tumors. It consists of different proportions of vascular, smooth muscle, and fat and has two types, namely, tuberous sclerosis complex (TSC)-associated and sporadic types (1-3). TSC is an autosomal dominant disease that affects multiple organs of the body. It is usually accompanied by the growth of hamartomas in the skin, brain, kidneys, lung, and heart. The various manifestations of TSC usually appear at different stages of life. In infancy, the most common manifestations are hamartomas, seizures, skin lesions, and rhabdomyosarcoma in the brain. TSC-associated RAML often develops at a younger age and grows much faster over time than sporadic RAML. Large RAML ( $>3-4 \mathrm{~cm}$ in diameter) may develop into an aneurysm, which may rupture, bleed, and

Correspondence to: $\mathrm{Mr}$. Zhengming $\mathrm{Su}$ or Professor Yongzhong He, Department of Urology, The Fifth Affiliated Hospital of Guangzhou Medical University, 621 Gangwan Road, Guangzhou, Guangdong 510700, P.R. China

E-mail: jaminsu@163.com

E-mail: hyzprc@163.com

Key words: tuberous sclerosis complex, renal angiomyolipoma become life-threatening, or it may compress the normal kidney tissue, leading to renal failure. Therefore, early detection, correct diagnosis, and reasonable treatment are very important for patients with RAML. We present a case of giant RAML and discussed the diagnosis and treatment of TSC-associated RAML.

\section{Case report}

The 20-year-old young female patient has been diagnosed with epilepsy since childhood and has been treated with valproate sustained release tablets. She was admitted to the department of neurology of our hospital due to repeated daze, occasionally accompanied by nausea, vomiting, and hallucinations. Physical examination showed that the patient was slightly slow in response and expression, and her calculation was poor. Multiple small papules and nodules can be seen on her cheeks. Palpation of the right upper abdomen revealed a tough mass with smooth surface, fixed position, and no tenderness. Valproic acid test in the laboratory showed that the patient's CYP2C9 genotype was CYP2C811, and the enzyme activity was fast metabolism (EM) type. POLG (A467TG $>$ A) was of wild homozygous type, POLG (W748SC $>$ G) wild homozygous type SLCO1B1 genotype was 1blb, and ApoE genotype was E2E4. The electrolyte, liver enzymes, renal functions, cardiac functions, routine examination of blood and urine, and coagulation functions were normal. Abdominal enhanced CT scan showed that the liver was obviously pushed, and the spine was scoliotic. A huge mass occupied the right upper quadrant, with a size of $236 \times 125 \times 149 \mathrm{~mm}$. The CT value was about-78HU. Blood vessels could be seen inside, and the boundary between the blood vessel and the kidney was unclear. The surrounding tissue was pushed, and the right kidney was displaced to the middle abdomen (Fig. 1). Moreover, two inhomogeneous enhancement nodules were present in the middle and lower pole of the right kidney. The larger nodule (26x33 mm in size) was in the right kidney. Multiple low-density shadows with no enhancement were also evident in the right kidney. In the left kidney, an inhomogeneous enhancement nodule was present on the upper pole, and its size was $28 \times 29 \mathrm{~mm}$. Multiple no enhancement low-density shadows (Fig. 2) were distributed in the left kidney. The right adrenal gland was unclear. The shape, size, and density of the left adrenal gland were normal. No obvious dilation and hydrops were observed in the bilateral 
renal pelvis and calyces, and the intestinal curve was pushed. No obvious enlarged lymph nodes and no ascites signs were seen behind the peritoneum. The result of the Chest CT scan showed multiple nodular shadows with sizes of $\sim 2-3 \mathrm{~mm}$ in the right upper lobe and dorsal right lower lobe. A nodular shadow, $\sim 3 \mathrm{~mm}$ in size, was seen under the pleura of the tongue of the left upper lobe. Cranial MRI showed the following results. Abnormal signals were detected from the bilateral cerebral hemisphere cortex, right putamen, and right caudate nucleus. A slightly low signal was observed in T1WI. A slightly high signal was observed in T2WI and FRAIR. The DWI showed no diffusion with limited high signal. Under the bilateral maxillary sinus mucosa, round-like long $\mathrm{T} 1$ long $\mathrm{T} 2$ signal shadows were seen, and the larger one was $\sim 15 \times 17 \mathrm{~mm}$ in size. Considering the patient's medical history and the results of laboratory examination, the diagnosis included epilepsy, giant hamartoma of the right kidney, hamartoma of the left kidney, multiple pulmonary nodules, and abnormal head signals, which were highly consistent with the manifestations of TSC. Although the patient had no obvious symptoms of abdominal visceral compression, and laboratory examination showed no obvious abnormalities in the kidney function. The compression of a huge abdominal mass caused the liver and kidney to deviate significantly and led to spine scoliosis. The continued growth of the mass may affect the function of the kidney and the digestive system. Moreover, the overgrown mass puts the patient at risk of spontaneous rupture and bleeding, which are life-threatening. So, the mass was indicated for surgical resection. After comprehensive preparation before the operation, the patient underwent open mass resection and right kidney partial resection. The appearance of the tumor is shown below (Fig. 3). Postoperative pathological results (Fig. 4) confirmed RAML, and the immunohistochemical results were as follows: HMB45 +, SMA +, Des +, S100 +, and Ki67 (1\% + ) (Fig. 5). The patient recovered smoothly after the operation. No recurrence of kidney tumors was found. Nodules in the lungs and abnormal head signals remained the same after the half-year follow-up period.

\section{Discussion}

The diagnosis of RAML. RAML is the most common benign renal tumor. RAML can be divided into sporadic and tuberous sclerosis (TSC) related types. At present, RAML is mostly sporadic and occurs more commonly in women are than in men. It is usually first found by B-ultrasound and then diagnosed by CT or MRI. TSC is an autosomal dominant inherited disease caused by mutations in the TSC 1 gene on chromosome 9 or the TSC 2 gene on chromosome 16 (4). Mutation of TSC1 or TSC2 gene causes the abnormal function of the TSC1/TSC2 complex, releases inhibitory effect on mechanistic target of rapamycin complex 1 (mTORC1), promotes cell growth, and inhibits autophagy, leading to TSC. TSC affects multiple organs of the body and is usually accompanied by the growth of hamartomas in the skin, brain, kidney, lung, and heart. Fat-containing angiomyolipomas have been observed in $80 \%$ of TSC patients. Fat-poor lesions are also common in patients with TSC, but occur in $<0.1 \%$ of the general population. Renal manifestations in TSC include RAML and multiple renal cysts (5). Compared with sporadic
RAML, the tumor is multiple and tends to grow to a larger volume; it is more likely to rupture, which results in hemorrhagic shock secondary to hematuria or retroperitoneal bleeding. Angiomyolipomas in the kidney can cause serious bleeding issues because of its vascular nature and can necessitate dialysis and even renal transplantation. In sporadic RAML cases, the tumor is generally single and asymptomatic. The typical symptoms of TSC-related RAML include abdominal pain (53\%), palpable masses (47\%), and gross hematuria (23\%). These symptoms are typical in patients with multiple sclerosis whose tumor is larger than $4 \mathrm{~cm}$; this is called Lenk's triad. Other possible symptoms and signs include bleeding, nausea, vomiting, high blood pressure, anemia, fever, shock, and urinary tract infection. Children with TSC mutant genes have normal kidneys at birth, but renal cysts and RAML appear with age. TSC in the nervous system manifests as clinical symptoms related to the growth of hamartomas, such as cortical dysplasia, subependymal nodules, and subependymal giant cell astrocytoma (SEGA). Huge SEGA may block the flow of cerebrospinal fluid through the orifice, resulting in increased intracranial pressure, obstructive hydrocephalus, focal neurological deficit, and death. The growth of hamartomas and other disturbances to normal cell growth can cause epilepsy and neurological cognition, as well as behavioral and mental defects. Epilepsy occurs in $60-90 \%$ of TSC patients, usually starting within the first year of birth. Early-onset seizures or intractable seizures are associated with cognitive and learning disabilities. The remaining TSC neurological symptoms also include empirical cognitive impairment, autism, and other behavioral disorders (50\%) (6). Other manifestations of TSC include the following: Rhabdomyosarcoma of the heart; lymphangioleiomyomatosis, which is a common pulmonary manifestation of TSC with a particularly high incidence in women; retinopathy (multiple retinal hamartomas and retinal pigment spots); and skin lesions (e.g., angiofibroma, which eventually appears in $~ 90 \%$ of patients; depigmentation spots; nail fibroids; shark plaques; oral fibroids; and others) (7). In 2012, the International TSC Consensus Committee formulated two independent TSC diagnostic criteria, namely, clinical and genetic diagnoses. Clinical diagnosis depends on the 11 main features and six secondary features of the patient (Table I) (8), among which 2 major features or 1 major feature +2 or more minor features can be diagnosed as TSC. If only 1 major feature or only 2 minor features, then diagnosis is possible. The detection of TSC1 or TSC2 gene pathogenic mutations in non-lesioned tissues can be diagnosed as TSC. The use of peripheral blood samples for genetic testing is recommended, but negative genetic testing cannot exclude the diagnosis of TSC. In the present case, the patient presented with RAML, multiple renal cysts, epilepsy, mental retardation, facial hemangioma, multiple pulmonary nodules, and abnormal head signals. The abovementioned findings support clinical diagnosis.

The treatment of TSC-associated RAML. Early detection and diagnosis of RAML can reduce the occurrence of serious complications (9). mTOR inhibitors is a new type of treatment. Prior to the approval of everolimus in 2012, surgical resection or transarterial embolization was the standard treatment for 
Table I. Major and minor features of the tuberous sclerosis complex (7).

\section{Major features}

Hypomelanotic macules ( $\geq 3$, at least 5-mm diameter)

Angiofibromas $(\geq 3)$ or fibrous cephalic plaque

Ungual fibromas $(\geq 2)$

Shagreen patch

Multiple retinal hamartomas

Cortical dysplasias ${ }^{a}$

Subependymal nodules

Subependymal giant cell astrocytoma

Cardiac rhabdomyoma

LAM $^{\text {b }}$

Angiomyolipomas $(\geq 2)^{\mathrm{b}}$
Minor features

'Confetti' skin lesions

Dental enamel pits $(>3)$

Intraoral fibromas $(\geq 2)$

Retinal achromic patch

Multiple renal cysts

Non-renal hamartomas

${ }^{a}$ Includes tubers and cerebral white matter radial migration lines; ${ }^{b}$ a combination of the two major clinical features (LAM and angiomyolipomas) without other features. LAM, lymphangioleiomyomatosis.

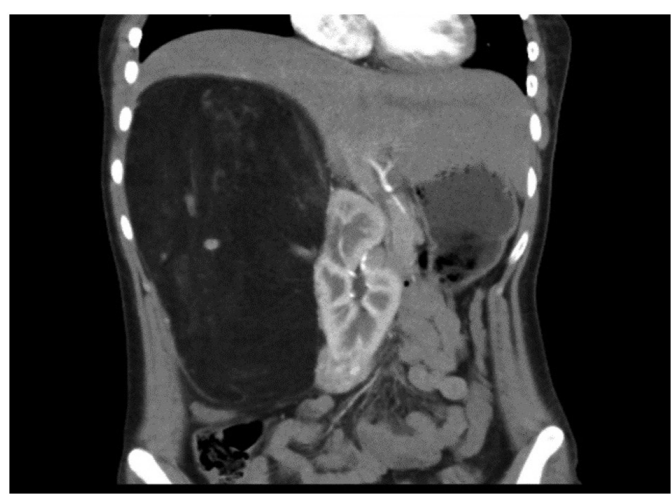

Figure 1. A huge mass in the right kidney. The surrounding tissue is squeezed.

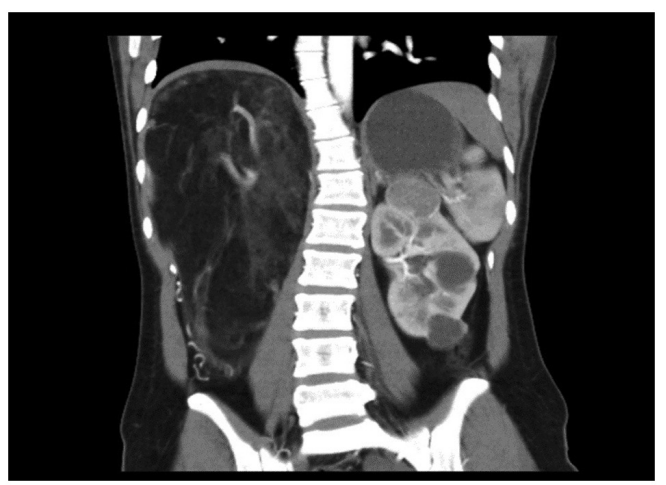

Figure 2. Multiple no enhancement low-density shadows are distributed in the left kidney.

most hamartomas requiring medical intervention. Excessive surgery resection may result in the loss of healthy kidney tissue and may damage the kidney, leading to the loss of its function. However, if the tumor tissue is not completely removed, the remaining tumor tissue may continue to grow, leading to recurrence $(10,11)$. Embolizing tumor blood vessels does not eliminate the root cause of TSC. The 2012 consensus

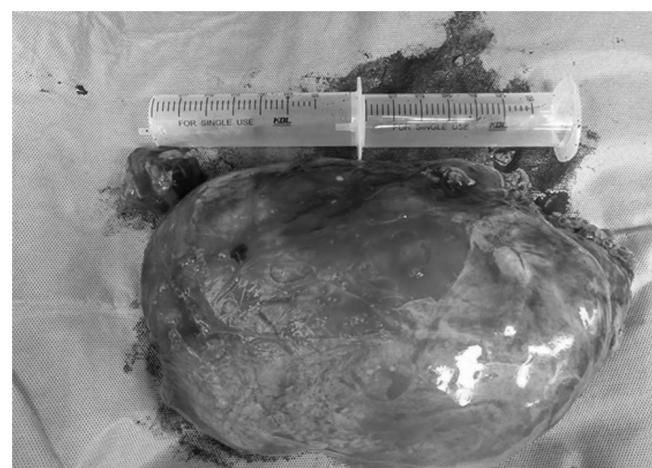

Figure 3. A giant tumor $(28 \times 15 \times 14 \mathrm{~cm})$ with a complete envelope, containing increased fat, blood vessels and smooth muscle tissue.

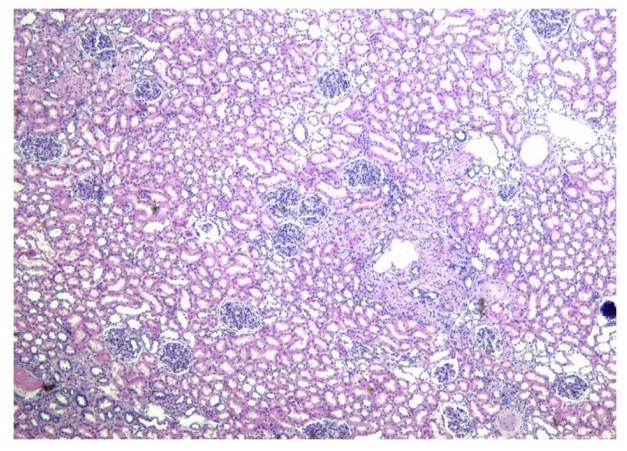

Figure 4. H\&E staining of tumor tissue (magnification, x100). A high quantity of vascular tissue, with scattered fat cells and smooth muscle is present $\mathrm{H} \& \mathrm{E}$, hematoxylin and eosin.

guidelines recommend mTOR inhibitors as the first-line treatment for asymptomatic RAML under $4 \mathrm{~cm}$ in diameter, whereas embolization and partial resection are used as second-line treatments. Everolimus is among the most commonly used mTOR inhibitors. Administration at $10 \mathrm{mg} / \mathrm{qd}$ oral treatment is recommended. This dosage can effectively reduce the volume of systemic AML and allow the non-invasive treatment of TSC 

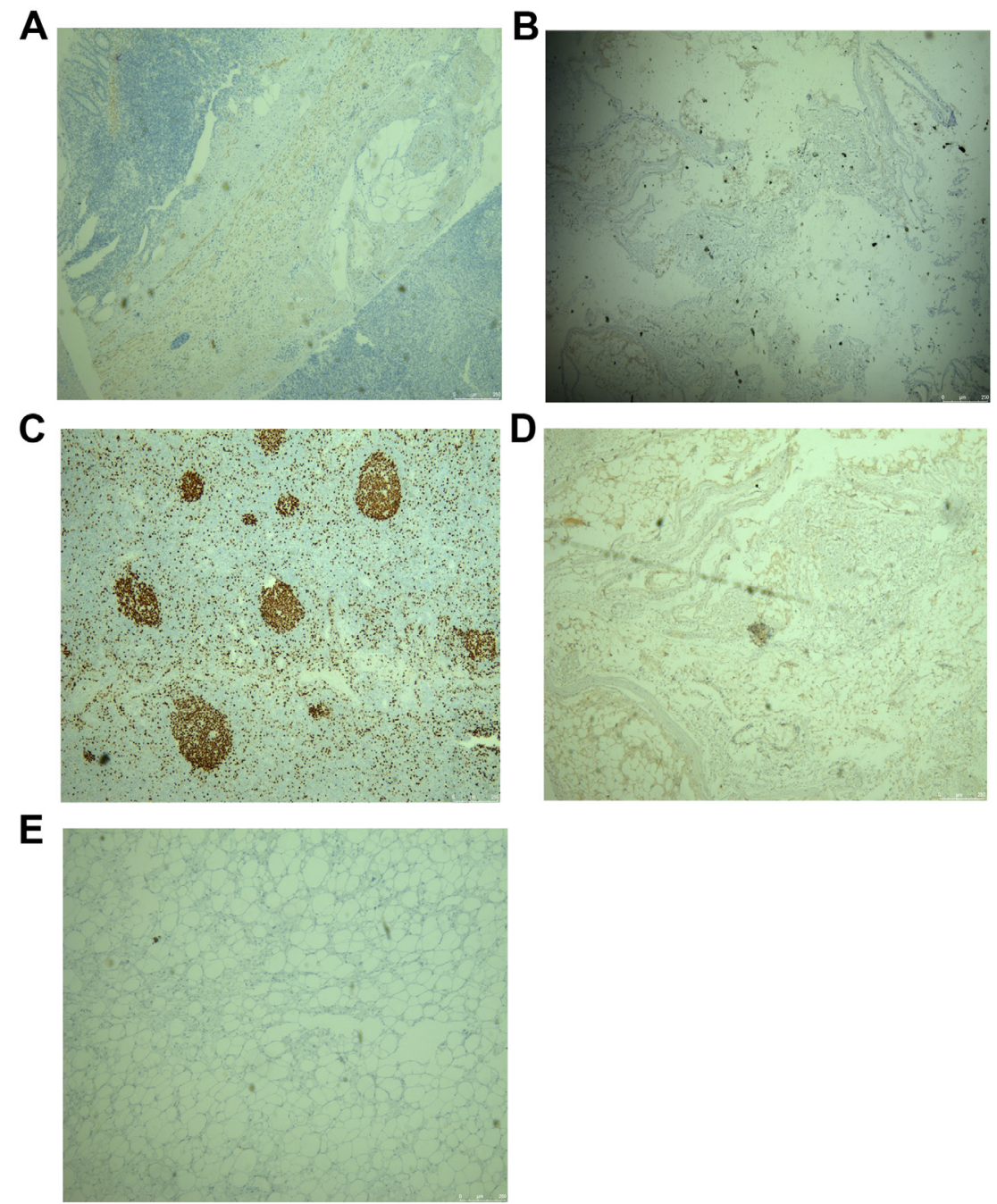

Figure 5. Immunohistochemical results (magnification, x100). (A) smooth muscle active positive, (B) Human melanoma black 45 positive, (C) Ki67 1\% positive, (D) S100 positive and (E) Desmin positive staining is presented.

patients, especially those with multiple disease manifestations. However, long-term treatment is usually required. The side effects associated with mTOR inhibitors and their management need to be known. These side effects include stomatitis/mucositis, respiratory infections, diarrhea, hypertriglyceridemia, hypercholesterolemia, bone marrow suppression, proteinuria, and arthralgia $(12,13)$. These adverse reactions are often related to drug concentration level, and dose adjustment may reduce the occurrence of adverse drug reactions. Interruption of the use of the drug and subsequent adjustment of the use of mTOR inhibitors may also help reduce systemic side effects (14). In the present case, the patient had no typical symptoms, such as typical hematuria and abdominal pain. Although the patient has not yet developed symptoms, such as hematuria, bleeding, nausea, vomiting, high blood pressure, anemia, fever, shock, and urinary tract infection, the large size of the tumor has caused scoliosis and pushing of the surrounding organs. With the passage of time and the continued growth of the tumor, the patient will likely show the abovementioned manifestations. Therefore, tumor resection is a reasonable treatment. Unfortunately, for some reason, this patient did not receive everolimus treatment. However, it is gratifying that after half a year of follow-up, the patient showed no obvious recurrence of
RAML. No progress in the lesions in pulmonary and intracranial areas was observed.

In a conclusion, clinical monitoring is essential for the treatment of TSC patients. The current guidelines recommend that for low-risk patients, especially asymptomatic minor patients with tumor diameters of less than $4 \mathrm{~cm}$, observation and waiting can be a suitable treatment method. Abdominal MRI or enhanced CT need to be performed every 1-3 years to evaluate the progress of RAML. Renal function and blood pressure need to be monitored at least once a year. Patients who do not take everolimus after selective arterial embolization or surgical intervention need to to undergo MRI or enhanced CT every 3-6 months, and their renal function and blood pressure need to be monitored. For patients with kidney disease, close follow-up and regular renal function monitoring are particularly important, because unlike other TSC manifestations, RAML and other kidney lesions may appear later in life and accumulate over time, thereby necessitating lifelong follow-up.

\section{Acknowledgements}

Not applicable. 


\section{Funding}

This work was supported by funding from the Medical Research Foundation of Guangdong Province of China (grant no. A2018093) and the Guangzhou Education Bureau Innovation Team Project (grant no. 201400902).

\section{Availability of data and materials}

The datasets used and/or analyzed during the present study are available from the corresponding author on reasonable request.

\section{Authors' contributions}

PS, YH and ZS acquired the data, performed the literature review and performed surgery and pre-operative administration. These authors also drafted, reviewed and edited the manuscript for intellectual content. ZS conceived the current study, analyzed the data and revised the manuscript for important intellectual content. PS performed surgery, analyzed data and images and edited the manuscript. SC performed pathological staining and pathological diagnosis. SC obtained radiological images and performed radiological diagnosis. All authors have read and approved the manuscript.

\section{Ethics approval and consent to participate}

The present case study was approved by the Ethics Committee of the Fifth Affiliated Hospital of Guangzhou Medical University of China. The patient provided consent for inclusion in this study.

\section{Patient consent for publication}

Written informed consent was obtained from the patient for the publication the case details and any associated images.

\section{Competing interests}

The authors declare that they have no competing interests.

\section{References}

1. Franz DN, Bissler JJ and McCormack FX: Tuberous sclerosis complex: Neurological, renal and pulmonary manifestations. Neuropediatrics 41: 199-208, 2010.

2. Curatolo P, Bombardieri R and Jozwiak S: Tuberous sclerosis. Lancet 372: 657-668, 2008.

3. Crino PB, Nathanson KL and Henske EP: The tuberous sclerosis complex. N Engl J Med 355: 1345-1356, 2006.
4. van Slegtenhorst M, de Hoogt R, Hermans C, Nellist M, Janssen B, Verhoef S, Lindhout D, van den Ouweland A, Halley D, Young J, et al: Identification of the tuberous sclerosis gene TSC1 on chromosome 9q34. Science 277: 805-808, 1997.

5. Kozłowska J and Okoń K: Renal tumors in postmortem material. Pol J Pathol 59: 21-25, 2008.

6. Krueger DA: Management of CNS-related disease manifestations in patients with tuberous sclerosis complex. Curr Treat Options Neurol 15: 618-633, 2013.

7. Samuels JA: Treatment of renal angiomyolipoma and other hamartomas in patients with tuberous sclerosis complex. Clin J Am Soc Nephrol 12: 1196-1202, 2017.

8. Northrup H and Krueger DA; International Tuberous Sclerosis Complex Consensus Group: Tuberous sclerosis complex diagnostic criteria update: Recommendations of the 2012 international tuberous sclerosis complex consensus conference. Pediatr Neurol 49: 243-254, 2013.

9. Wheless JW and Klimo P Jr: Subependymal giant cell astrocytomas in patients with tuberous sclerosis complex: Considerations for surgical or pharmacotherapeutic intervention. J Child Neurol 29: 1562-1571, 2014.

10. Krueger DA and Northrup H; International Tuberous Sclerosis Complex Consensus Group: Tuberous sclerosis complex surveillance and management: Recommendations of the 2012 international tuberous sclerosis complex consensus conference. Pediatr Neurol 49: 255-265, 2013.

11. Boorjian SA, Frank I, Inman B, Lohse CM, Cheville JC, Leibovich BC and Blute ML: The role of partial nephrectomy for the management of sporadic renal angiomyolipoma. Urology 70: 1064-1068, 2007.

12. Bissler JJ, McCormack FX, Young LR, Elwing JM, Chuck G, Leonard JM, Schmithorst VJ, Laor T, Brody AS, Bean J, et al: Sirolimus for angiomyolipoma in tuberous sclerosis complex or lymphangioleiomyomatosis. N Engl J Med 358: 140-151, 2008.

13. Lebwohl D, Thomas G, Lane HA, O'Reilly T, Escudier B, Yao JC, Pavel M, Franz D, Berg W, Baladi JF, et al: Research and innovation in the development of everolimus for oncology. Expert Opin Drug Discov 6: 323-338, 2011.

14. Sheth RA, Feldman AS, Paul E, Thiele EA and Walker TG: Angiographic and volumetric effects of mammalian target of rapamycin inhibitors on angiomyolipomas in tuberous sclerosis. World J Radiol 8: 308-315, 2016. 